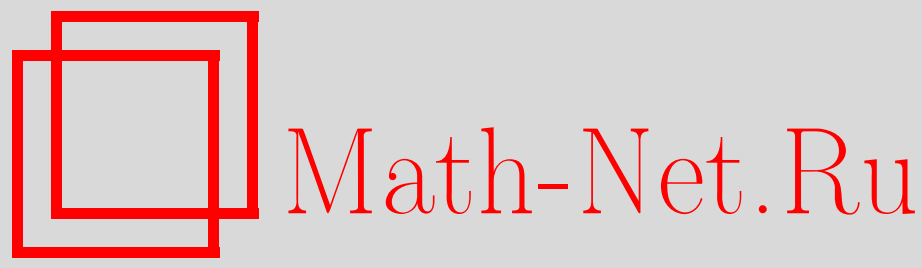

Ж. Зинн-Жустин, Важная роль функциональных интегралов в современной физике, ТМФ, 2011, том 169, номер 1, 20-31

DOI: https://doi.org/10.4213/tmf6705

Использование Общероссийского математического портала Math-Net.Ru подразумевает, что вы прочитали и согласны с пользовательским соглашением http: //www . mathnet.ru/rus/agreement

Параметры загрузки:

IP : 54.196 .121 .252

26 апреля 2023 г., 10:16:44

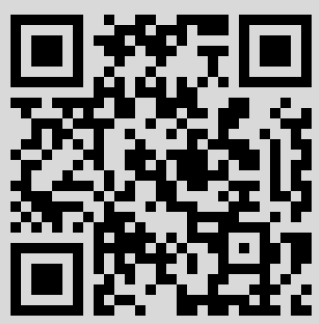




\title{
ВАЖНАЯ РОЛЬ ФУНКЦИОНАЛЬНЫХ ИНТЕГРАЛОВ В СОВРЕМЕННОЙ ФИЗИКЕ
}

\begin{abstract}
Двадцатое столетие явилось свидетелем возникновения физики флуктуирующих систем, как статистических, так и квантовых. Этим до некоторой степени объясняется важная роль, которую играют функциональные интегралы в современной физике. Здесь рассмотрено несколько ярких примеров физических задач, в которых решающим оказывается использование интегралов по траекториям и в более общем случае по полям.
\end{abstract}

Ключевые слова: флуктуирующие системы, статистическая механика, квантовая механика, функциональный интеграл.

\section{1. КЛАССИЧЕСКИЕ УРАВНЕНИЯ: ЗАГАДОЧНЫЙ ВАРИАЦИОННЫЙ ПРИНЦИП}

1.1. Ньютоновская механика. Следуя догадкам Мопертюи [1] и работам Эйлера [2] и Лагранжа, развившим точную формулировку вариационного принципа, Лагранж [3] показал, что уравнения движения ньютоновской механики можно вывести из вариационного принципа. Это направление достигло кульминации в гамильтоновом принципе [4], согласно которому уравнения движения для широкого класса (голономных) систем классической механики можно вывести из математической величины - интеграла действия, или лагранжиана

$$
\mathcal{A}(q)=\int_{t^{\prime}}^{t^{\prime \prime}} d t \mathcal{L}(\mathbf{q}(t), \dot{\mathbf{q}}(t) ; t),
$$

формулируя условия стационарности действия по отношению к вариациям траектории $\mathbf{q}(t)$. Получающиеся уравнения

$$
\delta \mathcal{A}=0 \quad \Rightarrow \quad \frac{d}{d t} \frac{\partial \mathcal{L}}{\partial \dot{q}_{i}}=\frac{\partial \mathcal{L}}{\partial q_{i}}
$$

принимают вид, получивший название уравнений Эйлера-Лагранжа.

Заметим, что в этих рамках действие и лагранжиан являются чисто математическими величинами.

${ }^{*}$ CEA-IRFU and Institut de Physique Théorique, Centre de Saclay, Gif-sur-Yvette, France. E-mail: zinn@dapnia.cea.fr 
1.2. Частица в магнитном поле. Хотя исходно вариационный принцип был доказан только в применении к классической механике, впоследствии было обнаружено, что уравнения движения частицы в статическом магнитном поле $\mathbf{B}$, которые имеют вид

$$
m \ddot{\mathbf{q}}=e \dot{\mathbf{q}} \times \mathbf{B}(\mathbf{q}), \quad \nabla \cdot \mathbf{B}(\mathbf{q})=0,
$$

также можно, что совершенно замечательно, вывести из некоторого принципа действия при условии, что вводится дополнительная математическая величина - вектор-потенциал

$$
\mathbf{B}(\mathbf{q})=-\nabla \times \mathbf{A}(\mathbf{q}) .
$$

Лагранжиан тогда можно записать как

$$
\mathcal{L}(\mathbf{q}, \dot{\mathbf{q}})=\frac{1}{2} m \dot{\mathbf{q}}^{2}-e \mathbf{A}(\mathbf{q}) \cdot \dot{\mathbf{q}} .
$$

В этом классическом формализме вектор-потенциал не рассматривается как физическая величина, поскольку он определен только с точностью до градиента. Действительно, вектор-потенциалы, связанные калибровочным преобразованием

$$
\mathbf{A}(\mathbf{q}) \mapsto \mathbf{A}(\mathbf{q})+\nabla \Omega(\mathbf{q})
$$

являются эквивалентными.

1.3. Электромагнетизм и уравнения Максвелла. Уравнения Максвелла (в вакууме) можно записать как

$$
\begin{array}{ll}
\nabla \cdot \mathbf{E}=\rho, & \nabla \times \mathbf{B}-\frac{\partial \mathbf{E}}{\partial t}=\mathbf{J}, \\
\nabla \cdot \mathbf{B}=0, & \nabla \times \mathbf{E}+\frac{\partial \mathbf{B}}{\partial t}=0,
\end{array}
$$

где $\mathbf{E}$ и $\mathbf{B}$ - электрическое и магнитное поля, а $\rho$ и $\mathbf{J}-$ заряд и плотность тока.

В квадри-ковариантных обозначениях

$$
t \equiv x_{0}, \quad F_{i 0}=E_{i}, \quad F_{i j}=\sum_{k} \epsilon_{i j k} B_{k}, \quad J_{0}=\rho, \quad i, j=1,2,3,
$$

два первых уравнения (1) сводятся к

$$
\sum_{\mu=0}^{3} \partial_{\mu} F^{\mu \nu}=J^{\nu} \Rightarrow \sum_{\nu=0}^{3} \partial_{\nu} J^{\nu}=0 .
$$

Из двух оставшихся уравнений $(1)$ следует, что тензор $F_{\mu \nu}$ выражается через вектор-потенциал или калибровочное поле $A_{\mu}(x)$ как

$$
F_{\mu \nu}=\partial_{\mu} A_{\nu}-\partial_{\nu} A_{\mu}
$$

Здесь снова калибровочное поле не является непосредственно физическим, будучи определенным только с точностью до абелева калибровочного преобразования

$$
A_{\mu}(x) \mapsto A_{\mu}(x)+\partial_{\mu} \Omega(x)
$$


Замечательно, что с введением этой новой математической величины уравнения Максвелла оказалось возможным вывести из принципа действия с плотностью лагранжиана

$$
\mathcal{L}(\mathbf{A}, \dot{\mathbf{A}})=-\frac{1}{4} \sum_{\mu, \nu} F^{\mu \nu} F_{\mu \nu}-\sum_{\mu} J^{\mu} A_{\mu}, \quad F_{\mu \nu} \equiv \partial_{\mu} A_{\nu}-\partial_{\nu} A_{\mu}
$$

и калибровочно-инвариантным действием

$$
\mathcal{A}=\int d^{4} x \mathcal{L}(\mathbf{A}, \dot{\mathbf{A}}) .
$$

1.4. Общая теория относительности. В релятивистской теории гравитации Эйнштейна (или общей теории относительности) уравнения движения также можно вывести из принципа действия.

Например, в отсутствие материи в терминах метрического тензора $g_{\mu \nu}(x)$ они имеют вид

$$
R_{\mu \nu}(\mathbf{g}(x))-\frac{1}{2} R(\mathbf{g}(x)) g_{\mu \nu}=0
$$

где $R$ - скалярная кривизна, а $R_{\mu \nu}$ - тензор Риччи. Эти уравнения можно вывести, варьируя действие Эйнштейна-Гильберта [5]

$$
\mathcal{A}(\mathbf{g})=\int d^{4} x(-g(x))^{1 / 2} R(\mathbf{g}(x)),
$$

где $g(x)$ - определитель метрического тензора. Вариационное свойство по-прежнему выполнено в присутствии материи и космологической постоянной.

\section{5. Интеграл по траекториям в квантовой механике и классическом} пределе. Теперь возникает вопрос: почему все фундаментальные классические уравнения движения можно вывести как условие стационарности локального действия? На первый взгляд, квантовая механика в ее гамильтоновой формулировке не дает прямого ответа. Первостепенным достижением квантовой механики в формулировке интеграла по траекториям (и квантовой теории поля в формулировке интеграла по полям) следует считать тот факт, что такая формулировка дает непосредственное объяснение этого свойства. Согласно Фейнману [6] квантовая эволюция дается интегралом по траекториям вида (см., например, [7], [8])

$$
\left\langle q^{\prime \prime}\left|U\left(t^{\prime \prime}, t^{\prime}\right)\right| q^{\prime}\right\rangle=\mathcal{N} \int_{q\left(t^{\prime}\right)=q^{\prime}}^{q\left(t^{\prime \prime}\right)=q^{\prime \prime}}[d q(t)] e^{i \mathcal{A}(q) / \hbar}
$$

где $U\left(t^{\prime \prime}, t^{\prime}\right)$ - оператор эволюции, $\mathcal{A}=\int d t \mathcal{L}$ - классическое действие (интеграл по времени от классического лагранжиана), суммирование выполняется по всем возможным траекториям $q(t)$, удовлетворяющим граничным условиям при временах $t^{\prime}$ и $t^{\prime \prime}$.

В классическом пределе, при $\hbar \rightarrow 0$, интеграл по траекториям можно вычислить методом стационарной фазы, и оказывается, что основной вклад в него вносят пути, которые оставляют действие стационарным: это в точности классические пути. Это свойство обобщается на релятивистскую квантовую теорию поля. Интегралы по траекториям при этом заменяются на интегралы по полям. Поэтому формулировка 
в рамках интегралов по траекториям (полям) дает более наглядную картину квантовой механики, придавая непосредственный смысл понятию квантовых флуктуаций, позволяет понять вариационные свойства классических уравнений и, наконец, дает удобное средство для вычисления квантовых поправок.

1.6. Квантовая гравитация. В качестве нетривиального следствия мы приходим к догадке, что поскольку классические уравнения общей теории относительности следуют из вариационного принципа, интеграл по полям метрики (или, в общем случае, спиновой связности) от выражения $e^{i \mathcal{S}_{\mathrm{EH}} / \hbar}$, куда входит действие Эйнштейна-Гильберта $\mathcal{S}_{\mathrm{EH}}$, при должной регуляризации на малых расстояниях (что, разумеется, представляет собой нетривиальный вопрос) должен иметь непосредственное отношение к квантовой гравитации. Действие Эйнштейна-Гильберта является, судя по всему, одним из первых членов в разложении некоторого эфбективного квантового действия.

\section{2. УНИТАРНОСТЬ И КОВАРИАНТНОСТЬ РЕЛЯТИВИСТСКОЙ КВАНТОВОЙ ТЕОРИИ ПОЛЯ}

Стандартная гамильтонова формулировка релятивистской квантовой теории поля является явно унитарной, но не явно ковариантной. Напротив, как впервые заметил Дирак [9], матричные элементы соответствующего оператора эволюции для инфинитезимального интервала времени включают в себя лагранжиан, а потому являются явно ковариантными. Интегралы по траекториям позволяют обобщить этот результат на конечные временны́е интервалы. Доказательство основано на следующем рассуждении.

В квантовой механике, что вполне естественно, представление матричных элементов оператора эволюции $U\left(t^{\prime \prime}, t^{\prime}\right)$ между временами $t^{\prime}$ и $t^{\prime \prime}$ в виде интеграла по траекториям имеет вид

$$
\left\langle q^{\prime \prime}\left|U\left(t^{\prime \prime}, t^{\prime}\right)\right| q^{\prime}\right\rangle=\int_{q\left(t^{\prime}\right)=q^{\prime}}^{q\left(t^{\prime \prime}\right)=q^{\prime \prime}}[d p(t) d q(t)] e^{i \mathcal{A}(p, q) / \hbar},
$$

где $p$ и $q$ - переменные фазового пространства (координата и сопряженный импульс), а $\mathcal{A}(p, q)$ - классическое действие в гамильтоновом формализме:

$$
\mathcal{A}(p, q)=\int_{t^{\prime}}^{t^{\prime \prime}}[p(t) \dot{q}(t)-H(p(t), q(t) ; t)] d t .
$$

В таком виде выражается решение уравнения Шредингера и тем самым имеется явная согласованность с унитарностью. Когда классический гамильтониан $H$ представляет собой квадратичную форму по $p$, вида $p^{2} /(2 m)+V(q)$, интеграл по $p$ является гауссовым и его можно вычислить явно:

$$
\int[d p(t)] \exp \left(\frac{i}{\hbar} \int_{t^{\prime}}^{t^{\prime \prime}} d t\left(p(t) \dot{q}(t)-p^{2}(t) /(2 m)\right)\right) \propto \exp \left(\frac{i}{\hbar} \int_{t^{\prime}}^{t^{\prime \prime}} d t \frac{1}{2} m \dot{q}^{2}(t)\right) .
$$

Интегрирование сводится к замене $p(t)$ на решение $m \dot{q}(t)$ классического уравнения и таким образом порождает лагранжиан. При этом воспроизводится выражение (2):

$$
\left\langle q^{\prime \prime}\left|U\left(t^{\prime \prime}, t^{\prime}\right)\right| q^{\prime}\right\rangle=\int_{q\left(t^{\prime}\right)=q^{\prime}}^{q\left(t^{\prime \prime}\right)=q^{\prime \prime}}[d q(t)] \exp \left(\frac{i}{\hbar} \int_{t^{\prime}}^{t^{\prime \prime}} d t \mathcal{L}(q, \dot{q})\right)
$$


где

$$
\mathcal{L}(q, \dot{q})=\frac{1}{2} m \dot{q}^{2}-V(q) .
$$

Этот метод обобщается на релятивистскую квантовую теорию: лагранжева формулировка является явно релятивистски ковариантной, в отличие от гамильтоновой формулировки, которая является явно унитарной.

\section{3. НЕАБЕЛЕВЫ КАЛИБРОВОЧНЫЕ ТЕОРИИ}

Интегралы по полям, обобщающие интегралы по траекториям на случай квантовых теорий поля, продемонстрировали свою исключительную полезность [10]-[12]. Например, они сыграли решающую роль в задаче о квантовании и перенормировке неабелевых калибровочных теорий.

3.1. Проблема квантования. Как и в квантовой электродинамике (абелевы калибровочные теории), из-за калибровочной инвариантности не все компоненты калибровочного поля $\mathbf{A}_{\mu}(x)$ являются динамическими, и непосредственное каноническое квантование невозможно. Однако, в отличие от квантовой электродинамики, квантование неабелевых калибровочных теорий, даже без материальных полей, не следует из простых эвристических аргументов. Прямое применение более непосредственных методов, которые работают в случае квантовой электродинамики, здесь невозможно [13].

Неабелевы калибровочные теории и интегралы по полям. Калибровочное поле $\mathbf{A}_{\mu}(x)$ преобразуется по присоединенному представлению группы $G$. Если оно представлено как элемент алгебры Ли $\mathcal{L}(G)$, а $\mathbf{g}(x)$ - элемент группы, зависящий от пространства-времени, то калибровочное преобразование имеет вид

$$
\mathbf{A}_{\mu}(x) \mapsto \mathbf{g}(x) \mathbf{A}_{\mu}(x) \mathbf{g}^{-1}(x)+\mathbf{g}(x) \partial_{\mu} \mathbf{g}^{-1}(x)
$$

В формализме интеграла по полям наивное представление эволюционного оператора в виде интеграла по полям

$$
U=\int\left[d \mathbf{A}_{\mu}\right] e^{i \mathcal{A}(\mathbf{A}) / \hbar}
$$

не определено, потому что действие $\mathcal{A}$, будучи калибровочно-инвариантным, не зависит от одного из полей интегрирования.

Необходимо проинтегрировать один раз по каждой калибровочной копии, т. е. ограничить интегрирование на сечение пространства калибровочных полей, пересекающее каждую калибровочную орбиту один раз (по крайней мере, в теории возмущений для слабых полей).

Типичное ковариантное сечение (калибровка Ландау) имеет вид

$$
\sum_{\mu} \partial_{\mu} \mathbf{A}^{\mu}(x)=0 .
$$

Непростую задачу в этом случае представляет собой определение меры на калибровочном сечении. Одно из решений основано на методе Фаддеева-Попова [14] и приводит к введению духов Фаддеева-Попова. Однако замечательно, и вполне типично для методов, связанных с интегралами по траекториям, что искомый метод можно получить из конечномерных интегралов [12]. 
3.2. Сечения и меры. Пусть $E_{\alpha}(\varphi)$ - множество гладких функций переменных $\varphi^{\alpha}$ таких, что $E_{\alpha}=E_{\alpha}(\varphi)$ - взаимно однозначное отображение. Обозначим через $\varphi_{\mathrm{s}}$ решение системы

$$
E_{\alpha}\left(\varphi_{\mathrm{s}}\right)=0,
$$

а через $E_{\alpha \beta}$ - матрицу $E_{\alpha \beta} \equiv \partial E_{\alpha} / \partial \varphi_{\beta}$. Для любой функции $F(\varphi)$ мы хотим вывести формальное выражение для $F\left(\varphi_{\mathrm{s}}\right)$. Начнем с тривиального тождества

$$
F\left(\varphi_{\mathrm{s}}\right)=\int\left\{\prod_{\alpha} d E^{\alpha} \delta\left(E_{\alpha}\right)\right\} F(\varphi(E)) .
$$

Теперь сделаем замену переменных $E \mapsto \varphi$. Якобиан равен $\operatorname{det} E_{\alpha \beta}$ :

$$
F\left(\varphi_{\mathrm{s}}\right)=\int\left\{\prod_{\alpha} d \varphi^{\alpha} \delta\left[E_{\alpha}(\varphi)\right]\right\} \operatorname{det} E_{\alpha \beta} F(\varphi) .
$$

Заменим $\delta$-функцию на ее представление Фурье:

$$
\prod_{\alpha} \delta\left[E_{\alpha}(\varphi)\right]=\int \prod_{\alpha} \frac{d \bar{\varphi}^{\alpha}}{2 i \pi} \exp \left(-\sum_{\alpha} \bar{\varphi}^{\alpha} E_{\alpha}(\varphi)\right)
$$

( $\bar{\varphi}$ является чисто мнимым). Любой определитель можно записать в виде интеграла по грассмановым переменным $\bar{c}^{\alpha}$ и $c^{\alpha}$ (идея, вытекающая из обобщения интеграла по траекториям на фермионы):

$$
\operatorname{det} E_{\alpha \beta}=\int \prod_{\alpha}\left(d \bar{c}^{\alpha} d c^{\alpha}\right) \exp \left(\sum_{\alpha, \beta} c^{\alpha} E_{\alpha \beta} \bar{c}^{\beta}\right) .
$$

Тогда выражение принимает вид

$$
F\left(\varphi_{\mathrm{s}}\right) \propto \int \prod_{\alpha}\left(d \varphi^{\alpha} d \bar{\varphi}^{\alpha} d \bar{c}^{\alpha} d c^{\alpha}\right) F(\varphi) e^{-S(\varphi, \bar{\varphi}, c, \bar{c})},
$$

где

$$
S(\varphi, \bar{\varphi}, c, \bar{c})=\sum_{\alpha} \bar{\varphi}^{\alpha} E_{\alpha}(\varphi)-\sum_{\alpha, \beta} c^{\alpha} E_{\alpha \beta}(\varphi) \bar{c}^{\beta} .
$$

Это выражение обладает фермионо-подобной симметрией, БРСТ-симметрией [15]: оно инвариантно относительно вариаций [12]

$$
\begin{aligned}
\delta \varphi^{\alpha} & =\varepsilon \bar{c}^{\alpha}, & & \delta \bar{c}^{\alpha}=0, \\
\delta c^{\alpha} & =\varepsilon \bar{\varphi}^{\alpha}, & \delta \bar{\varphi}^{\alpha} & =0,
\end{aligned}
$$

где $\varepsilon$ - грассманова постоянная. Обобщением БРСТ-симметрии является суперсимметрия.

В неабелевых калибровочных теориях переменные $\varphi_{\alpha}$ параметризуют калибровочную группу преобразований $\mathbf{g}(x)$, уравнения $E_{\alpha}=0$ определяют калибровочное сечение, $c^{\alpha}, \bar{c}^{\alpha}$ - духи Фаддеева-Попова [14], а $S$ становится дополнительным (не являющимся калибровочно-инвариантным) вкладом в квантованное калибровочное действие. Обобщенные калибровки [16] получаются путем рассмотрения стохастических уравнений [12].

Перенормировка. Все доказательства перенормируемости неабелевых калибровочных теорий также основаны на функциональных методах; в первых доказательствах [17] использовались тождества Славнова-Тейлора [18], однако БРСТ-симметрия [15] существенно упростила доказательство перенормируемости [19]. 


\section{4. КВАНТОВАНИЕ НЕЛИНЕЙНОЙ $\sigma$-МОДЕЛИ}

Нелинейная $\sigma$-модель [20] является квантовой теорией поля с глобальной $O(N)$ симметрией и $N$-компонентным скалярным полем $\phi(x)$, живущим на сфере $S_{N-1}$ :

$$
\phi^{2}(x)=1 .
$$

В терминах поля $\phi$ действие принимает вид свободного полевого действия:

$$
\mathcal{S}(\phi)=\frac{1}{2 g} \int d^{d} x\left[\partial_{\mu} \phi(x)\right]^{2},
$$

однако наличие связи приводит к взаимодействию. В рамках пертурбативного разложения $O(N)$-симметрия реализуется в фазе спонтанного нарушения симметрии и динамические поля соответствуют голдстоуновским модам. Первые (однопетлевые) вычисления, казалось, указывали на то, что $O(N)$-симметрия явно нарушается пертурбативными поправками. Позже в рамках канонической формулировки путем сложных вычислений было показано, что на самом деле слагаемые, приводящие к этим нарушениям, сокращаются.

Однако представление интегралов по полям дало правильный квантованный вид для всех порядков [21],

$$
\mathcal{Z}=\int[d \phi] \prod_{x} \delta\left(\phi^{2}(x)-1\right) e^{-\mathcal{S}(\phi) / g},
$$

а также геометрическую интерпретацию исходной ошибки: в рамках представления интегралов по полям она была эквивалентна замене $O(N)$-инвариантной меры на плоскую евклидову меру.

Более того, чтобы придать смысл модели вне рамок теории возмущений, можно ввести решеточную регуляризацию, что приводит к $O(N)$-решеточной спиновой модели. Таким способом можно установить соотношение между нелинейной $\sigma$-моделью и статистической $\left(\phi^{2}\right)^{2}$-теорией поля.

\section{5. КРИТИЧЕСКИЕ ЯВЛЕНИЯ, КВАНТОВАЯ ТЕОРИЯ ПОЛЯ И РЕГУЛЯРИЗАЦИЯ}

Формулировка квантовой теории поля в рамках представления интегралов по полям подчеркивает ее связь с классической статистической физикой.

5.1. Критические явления. Следуя Вильсону, было осознано, что универсальные критические свойства широкого класса статистических моделей можно описывать с помощью евклидовой квантовой или статистической теории поля. Например, критические свойства $d$-мерной модели Изинга

$$
\mathcal{Z}=\sum_{\left\{S_{i}\right\}= \pm 1} \exp \left(J \sum_{i, j \text { n.n. }} S_{j}\right),
$$

где $\sum_{i, j \text { n.n. }}$ означает суммирование по ближайшим соседям, описываются квантовой $\phi^{4}$-теорией поля (в мнимом времени)

$$
\mathcal{Z}_{\phi^{4}}=\int[d \phi] e^{-\mathcal{S}(\phi)},
$$


где проводится интегрирование по всем полям $\phi(x), x \in \mathbb{R}^{d}$, a $\mathcal{S}(\phi)$ - евклидово действие

$$
\mathcal{S}(\phi)=\int d^{d} x\left[\frac{1}{2} \sum_{\mu}\left(\partial_{\mu} \phi(x)\right)^{2}+\frac{1}{2} \phi^{2}(x)+\frac{1}{4 !} g \phi^{4}(x)\right] .
$$

Это свойство обобщается на классические спиновые модели с $O(N)$-симметрией следующим образом:

$$
\mathcal{Z}=\sum_{\left\{\left|\mathbf{S}_{i}\right|\right\}=1} \exp \left(J \sum_{i, j \text { n.n. }} \mathbf{S}_{i} \cdot \mathbf{S}_{j}\right)
$$

что приводит к $\left(\phi^{2}\right)^{2}$-теории поля. Кроме того, техника интегралов по траекториям позволяет непосредственно доказать, что формальный предел $N=0$ описывает статистические свойства полимеров. При этом для вычисления универсальных критических свойств классических статистических систем вблизи непрерывного фазового перехода следует использовать ренормгрупповой подход в квантовой теории поля. Наоборот, относительно статистических моделей можно считать, что они дают регуляризацию на малых расстояниях в квантовой теории поля, которая без этого является некорректно определенной.

5.2. Численное моделирование в квантовой теории поля. Соответствие между классическими статистическими моделями и квантовой теорией поля позволило применить статистические методы к непертурбативному исследованию квантовых теорий поля. Мысль состояла в том, чтобы заменить континуальный интеграл по полям на решеточную регуляризацию. Тогда становятся доступными непертурбативные численные методы, такие как разложение сильной связи или моделирование типа моделирования методом Монте-Карло.

Замечательным примером является квантовая хромодинамика. В отсутствие материи моделирование основано на вильсоновской статсумме по плакетам [22]:

$$
\mathcal{Z}=\int \prod_{\text {links }\{i j\}} d \mathbf{U}_{i j} e^{-\beta_{p} \mathcal{S}(\mathbf{U})}, \quad \mathcal{S}(\mathbf{U})=-\sum_{\text {plaquettes }} \operatorname{tr} \mathbf{U}_{i j} \mathbf{U}_{j k} \mathbf{U}_{k l} \mathbf{U}_{l i},
$$

где $\mathbf{U}_{i j}$ - элемент группы, ассоциированный с ребрами, а $\mathcal{S}$ - плакетное действие. Решеточная формулировка также дает непертурбативное определение квантовой хромодинамики.

\section{6. ИНСТАНТОНЫ, ПОВЕДЕНИЕ В ВЫСОКИХ ПОРЯДКАХ И СУММИРУЕМОСТЬ ПО БОРЕЛЮ}

В квантовой теории поля поведение пертурбативного разложения в высоких порядках связано с неустойчивостью вакуума. Предложение применять интегралы по полям и инстантоны [23] позволило определить поведение пертурбативного разложения в высоких порядках в ряде квантовых теорий поля.

Важный вывод таков: ряды теории возмущений всегда расходятся. Более того, знание поведения в высоких порядках имеет непосредственные следствия для проблемы суммируемости по Борелю. 
6.1. Суммирование и применение к критическим индексам. Важным применением явилось использование суммирования пертурбативных разложений в $\left(\phi^{2}\right)^{2}$-квантовой теории поля для точного вычисления критических индексов (см. таблицу). Было показано, что метод, основанный на преобразовании и отображении Бореля, является очень надежным.

Точные критические индексы в $O(N)$-симметричной $\left(\phi^{2}\right)_{3}^{2}$-теории поля [24]

\begin{tabular}{|c|c|c|c|c|}
\hline$N$ & 0 & 1 & 2 & 3 \\
\hline$\tilde{g}^{*}$ & $1.413 \pm 0.006$ & $1.411 \pm 0.004$ & $1.403 \pm 0.003$ & $1.390 \pm 0.004$ \\
\hline$g^{*}$ & $26.63 \pm 0.11$ & $23.64 \pm 0.07$ & $21.16 \pm 0.05$ & $19.06 \pm 0.05$ \\
\hline$\gamma$ & $1.1596 \pm 0.0020$ & $1.2396 \pm 0.0013$ & $1.3169 \pm 0.0020$ & $1.3895 \pm 0.0050$ \\
\hline$\nu$ & $0.5882 \pm 0.0011$ & $0.6304 \pm 0.0013$ & $0.6703 \pm 0.0015$ & $0.7073 \pm 0.0035$ \\
\hline$\eta$ & $0.0284 \pm 0.0025$ & $0.0335 \pm 0.0025$ & $0.0354 \pm 0.0025$ & $0.0355 \pm 0.0025$ \\
\hline$\beta$ & $0.3024 \pm 0.0008$ & $0.3258 \pm 0.0014$ & $0.3470 \pm 0.0016$ & $0.3662 \pm 0.0025$ \\
\hline$\alpha$ & $0.235 \pm 0.003$ & $0.109 \pm 0.004$ & $-0.011 \pm 0.004$ & $-0.122 \pm 0.010$ \\
\hline$\omega$ & $0.812 \pm 0.016$ & $0.799 \pm 0.011$ & $0.789 \pm 0.011$ & $0.782 \pm 0.0013$ \\
\hline$\omega \nu$ & $0.478 \pm 0.010$ & $0.504 \pm 0.008$ & $0.529 \pm 0.009$ & $0.553 \pm 0.012$ \\
\hline
\end{tabular}

6.2. Потенциалы с вырожденными минимумами. В случае потенциалов с дискретными вырожденными классическими минимумами поведение в высоких порядках (полученное из инстантонного анализа) указывает на то, что пертурбативное разложение не является суммируемым по Борелю и, как следствие, не определяет однозначные функции. В простой квантовой механике с аналитическими потенциалами задачу можно исследовать систематически и показать, что следует учитывать все многоинстантонные конфигурации и вводить обобщенную процедуру суммирования [25], [26].

\section{7. КЛАССИЧЕСКАЯ И КВАНТОВАЯ СТАТИСТИЧЕСКАЯ ФИЗИКА}

Для скалярного квантового поля $\phi$ в $d$-мерном пространстве при температуре $T=1 / \beta$ квантовая статсумма имеет вид

$$
\mathcal{Z}=\int[d \phi] \exp \left(-\int_{0}^{\beta} d \tau \int d^{d} x \mathcal{S}(\phi)\right),
$$

где $\mathcal{S}$ - евклидово (в мнимом времени) действие, а бозе-поля удовлетворяют периодическим граничным условиям $\phi(0, x)=\phi(\beta, x)$. Однако представление интегралов по полям позволяет показать, что ту же статсумму можно интерпретировать как классическую статсумму в $(d+1)$-мерном пространстве с конечным размером $\beta$ и периодическими граничными условиями в одном пространственном направлении.

Это наблюдение имеет важные следствия для теории непрерывных фазовых переходов: оно связывает классические переходы в $(d+1)$-мерном пространстве с квантовыми переходами при нулевой температуре $(\beta=\infty)$ в $d$-мерном пространстве.

В более общем смысле взаимосвязь между классической и квантовой статистической физикой переводит квантовые эффекты при конечной температуре в эффекты 
конечных размеров в классической теории. Тогда эти эффекты можно исследовать ренормгрупповыми методами.

Конечные температуры и размерная редукция. В частности, при такой постановке высокие температуры ассоциируются с размерной редукцией. С технической точки зрения периодическое поле разлагается по модам Фурье (Мацубары):

$$
\phi(t, x)=\sum_{\nu} e^{i 2 \pi \nu t / \beta} \phi_{\nu}(x)
$$

При высоких температурах, вблизи непрерывного фазового перехода, когда корреляционная длина много больше температурной длины волны $\hbar \sqrt{2 \pi / m T}$, только нулевая мода является критической. Тогда можно интегрировать пертурбативно по всем ненулевым модам:

$$
e^{-\mathcal{S}_{\mathrm{eff}}\left(\phi_{0}\right)}=\int \prod_{\nu \neq 0}\left[d \phi_{\nu}\right] e^{-\mathcal{S}(\phi)} \quad \text { при } \quad \mathcal{Z}=\int\left[d \phi_{0}\right] e^{-\mathcal{S}_{\mathrm{eff}}\left(\phi_{0}\right)},
$$

однако при этом нулевая мода $\phi_{0}$ должна рассматриваться непертурбативно.

Разреженный (тем самым слабо взаимодействующий) бозе-газ. В качестве примера предложенные методы применим к разреженному бозе-газу, который описывается бозе-эйнштейновским конденсатом, возмущенным слабым отталкивающим взаимодействием. Исходный интеграл по полям $\psi^{*}, \psi$, периодическим по евклидову времени, имеет вид

$$
\mathcal{Z}=\int\left[d \psi(t, x) d \psi^{*}(t, x)\right] e^{-\mathcal{S}\left(\psi^{*}, \psi\right) / \hbar}
$$

Поскольку нас интересуют только длинноволновые явления, двухчастичный потенциал можно заменить $\delta$-функцией и параметризовать в терминах длины рассеяния $s$-волн $a$ (положительной, поскольку взаимодействие считается отталкивающим).

Тогда при $d=3$ эффективное евклидово действие системы можно записать как

$$
\begin{aligned}
\mathcal{S}\left(\psi^{*}, \psi\right)=- & \int_{0}^{\beta} d t \int d^{3} x\left[\psi^{*}(t, x)\left(\hbar \frac{\partial}{\partial t}+\frac{\hbar^{2}}{2 m} \nabla_{x}^{2}+\mu\right) \psi(t, x)+\right. \\
& \left.+\frac{2 \pi \hbar^{2} a}{m}\left(\psi^{*}(t, x) \psi(t, x)\right)^{2}\right],
\end{aligned}
$$

где $\mu$ - химический потенциал. Редуцированная статсумма в главным порядке принимает вид интеграла по полям:

$$
\mathcal{Z}=\int[d \phi(x)] e^{-\mathcal{S}(\phi)}
$$

где

$$
\mathcal{S}(\phi)=\int\left\{\frac{1}{2}\left[\partial_{\mu} \phi(x)\right]^{2}+\frac{1}{2} r \phi^{2}(x)+\frac{u}{4 !}\left[\phi^{2}(x)\right]^{2}\right\} d^{d} x,
$$

a $r=-2 m$ Т $\mu$ и при $d=3$ имеем $u=96 \pi^{2} a / \lambda^{2}$.

Евклидово действие сводится к обычной $O(2)$-симметричной $\left(\phi^{2}\right)^{2}$-теории поля, которая также описывает универсальные свойства перехода в сверхтекучем гелии. 


\section{8. НЕПЕРТУРБАТИВНЫЕ МЕТОДЫ ПРИ БОЛЬШИХ $N$}

В квантовых теориях поля с $O(N)$ - или $U(N)$-симметриями и полями в векторном представлении физические величины можно вычислить в пределе больших $N$, что дает непертурбативные результаты. В главным порядке результаты можно получить, суммируя фейнмановские диаграммы, однако методы интегралов по полям являются гораздо более простыми и могут быть легко продолжены на высшие порядки по $1 / N$. Приложения включают исследование $\left(\phi^{2}\right)^{2}$-теории, модели Гросса-Неве и т. д.

Например, в скалярных $U\left(\phi^{2}\right)$-теориях поля отправной точкой является включение в интеграл по полю $\phi$ тождества

$$
1=\int[d \lambda d \rho] \exp \left(i \int d^{d} x \lambda(x)\left[\rho(x)-\phi^{2}(x)\right]\right),
$$

который в этом случае допускает гауссово интегрирование по $\phi$ (подробнее см., например, [27]).

\section{Список литературы}

[1] P. L. M. de Maupertuis, "Les Loix du mouvement et du repos déduites d'un principe métaphysique", Histoire de l'Académie Royale des Sciences et Belles-Lettres, Institut de France, Paris, 1746, 267-294.

[2] L. Euler, "Additamentum II", Methodus Inveniendi Lineas Curvas Maximi Minime Proprietate Gaudentes, Sive Solutis Problematis Isoperimetrici Latissimo Sensu Accepti, original Latin text, Bousquet, Lausanne, Geneva, 1774.

[3] J.-L. Lagrange, Mécanique analytique, Chez la Veuve Desaint, Paris, 1788; "Mémoire sur la théorie du mouvement des fluides", Oeuvres de Lagrange, 4, ed. J. A. Serret, Gauthier-Villars, Paris, 1867, 695-748.

[4] W. R. Hamilton, Phil. Trans. R. Soc. Lond., 124 (1834), 247-308; 125 (1835), 95-144.

[5] D. Hilbert, Gött. Nachr., 27 (1915), 395-407.

[6] R. P. Feynman, Rev. Mod. Phys., 20:2 (1948), 367-387.

[7] J. Zinn-Justin, Scholarpedia, 4:2 (2009), 8674.

[8] Зинн-Жюстен Ж., Континуальный интеграл в квантовой механике, Физматлит, М., 2007.

[9] P. A. M. Dirac, Physik. Z. Sowjetunion, 3 (1933), 64-72; J. Schwinger, Selected Papers on Quantum Electrodynamics, Dover, New York, 1958.

[10] A. N. Vassiliev, "Etudes des symétries brisées par des méthodes fonctionnelles (Notes redigees par P. Moussa)", Cargèse Lectures in Physics, 5, ed. D. Bessis, Gordon and Breach, New York, 1971, 239-303.

[11] А.Н. Васильев, Функииональные методы в квантовой теории поля и статистике, Изд-во ЛГУ, Л., 1976.

[12] J. Zinn-Justin, Quantum Field Theory and Critical Phenomena, Oxford Univ. Press, Oxford, 1989.

[13] R. P. Feynman, Acta Phys. Polon., 24 (1963), 697-722.

[14] L. D. Faddeev, V. N. Popov, Phys. Lett. B, 25:1 (1967), 29-30; L. D. Faddeev, Scholarpedia, 4:4 (2009), 7389.

[15] C. Becchi, A. Rouet, R. Stora, Commun. Math. Phys., 42:2 (1975), 127-162; Ann. Phys., 98:2 (1976), 287-321; И.В. Тютин, Калибровочная инвариантность в теории поля и статистической физике в операторной формулировке, Препринт ФИАН, 39, 1975; C. M. Becchi, C. Imbimbo, Scholarpedia, 3:10 (2008), 7135. 
[16] G. 't Hooft, Nucl. Phys. B, 33:1 (1971), 173-199.

[17] B. W. Lee, J. Zinn-Justin, Phys. Rev. D, 5:12 (1972), 3121-3137; 3137-3155; 3155-3160; Erratum 8:12 (1973), 4654-4654; 7:4 (1973), 1049-1056.

[18] А. А. Славнов, ТМФ, 10:2 (1972), 153-161; J. C. Taylor, Nucl. Phys. B, 33:2 (1971), 436-444; A. A. Slavnov, Scholarpedia, 3:10 (2008), 7119.

[19] J. Zinn-Justin, "Renormalization of gauge theories", Trends in Elementary Particle Theory, Lectures Notes in Physics, 37, eds. H. Rollnik, K. Dietz, Springer, Berlin, 1975, 1-39; Mod. Phys. Lett. A, 14:19 (1999), 1227-1235, arXiv: hep-th/9906115; Scholarpedia, 4:1 (2009), 7120 .

[20] M. Gell-Mann, M. Lévy, Nuovo Cimento, 16 (1960), 705-726.

[21] J. Honerkamp, K. Meetz, Phys. Rev. D, 3:8 (1971), 1996-1998.

[22] K. G. Wilson, Phys. Rev. D, 10:8 (1974), 2445-2459.

[23] Л. Н. Липатов, Писъма в ЖЭТФ, 25:2 (1977), 116-119.

[24] J. C. Le Guillou, J. Zinn-Justin, Phys. Rev. B, 21:9 (1980), 3976-3998; R. Guida, J. Zinn-Justin, J. Phys. A, 31:40 (1998), 8103-8121, arXiv: cond-mat/9803240.

[25] J. Zinn-Justin, Nucl. Phys. B, 192:1 (1981), 125-140; 218:2 (1983), 333-348.

[26] U.D. Jentschura, J. Zinn-Justin, J. Phys. A, 34:18 (2001), L253-L258; J. Zinn-Justin, U. D. Jentschura, Ann. Phys., 313:1 (2004), 197-267, arXiv: quant-ph/0501136; 313:2 (2004), 269-325.

[27] M. Moshe, J. Zinn-Justin, Phys. Rept., 385:3-6 (2003), 69-228, arXiv: hep-th/0306133. 